\title{
A Basic Study on Improving Ultrasound Images
}

\author{
Paulami Shah \\ Computer Engineering \\ Department, \\ Mukesh Patel School of \\ Technology Management \& \\ Engineering, NMIMS \\ University, \\ Mumbai, India.
}

\author{
Saurabh Malgaonkar \\ Computer Engineering \\ Department, \\ Mukesh Patel School of \\ Technology Management \& \\ Engineering, NMIMS \\ University, \\ Mumbai, India.
}

\author{
Tanay Parekhji \\ Computer Engineering \\ Department, \\ Mukesh Patel School of \\ Technology Management \& \\ Engineering, NMIMS \\ University, \\ Mumbai, India.
}

\author{
Rahul Chavan \\ Computer Engineering Department, \\ Mukesh Patel School of Technology Management \& Engineering, NMIMS University, \\ Mumbai, India.
}

\begin{abstract}
This proposed study method involves the implementation of Frost filter, Lee filter and SRAD filter on ultrasound images and a quantitative evaluation of peak signal-to-noise ratio (PSNR) and Pratt's figure of merit (FOM).
\end{abstract}

\section{Keywords}

Ultrasound images, image processing, image filtering, SRAD, speckle

\section{INTRODUCTION}

Ultrasound image is often preferred over other medical imaging modalities because it is non-ionizing, portable, low cost and provides real time image formation. There is, however, a drawback when it comes to using medical ultrasound imaging, in that, the quality of the image is generally corrupted. This corruption is caused by a form of locally correlated multiplicative noise called, 'speckle'.

Speckle is the main problem in ultrasound images which affects the diagnostic information. In this approach, various speckle reduction filtering techniques have been studied and implemented on different ultrasound images [3].This system presents multi-scale based adaptive SRAD method using wavelet decomposition. SRAD is derived by the instantaneous coefficient of variation (ICOV) in the same way as Lee filter into a partial differential equation (PDE).

\section{LITERATURE SURVEY}

The Lee Filter was created to reduce and/or remove speckle noise. However, edge and point features of radial imagery cannot be compromised. They have to be kept intact during the process. The Lee filter gives enhanced data by combining both, a linear speckle noise model and the Minimum Mean Square Error (MMSE) design approach [2]. The Frost filter depends upon an exponentially damped convolution kernel. This kernel is responsible for adapting to the region containing edges by exploiting local statistics [2]. Anisotropic diffusion has been widely used to reduce speckle noise from ultrasound images by iterative modification of the image via partial differential equation [4].

\section{DESIGN}

In speckle filtering, a kernel is placed over each pixel one after the other. When placed, a mathematical calculation is done at the said pixel. This filtering technique uses the pixel values under the kernel and replaces the central pixel value with the calculated value. In this way, the entire image is gradually covered by moving the kernel along the image. The result of applying the filter is a smoothing effect, and the visual appearance of the speckle is, thereby, reduced.

\subsection{Frost Filter and Diffusion}

The Frost filter takes an aggressive stand on achieving equilibrium between the averaging filter and all- pass filter. This equilibrium is achieved by the formation of an exponentially shaped filter kernel. It varies from a basic average filter to an identity filter on a point wise adaptive basis. It must be noted that the filter's response may vary with the coefficient of variation. With a low coefficient of variation, the filter tends to be more average like while in the high coefficient case, it remains sharp and does not have any averaging effects. A speckled image can commonly be quantified as:

$$
v_{1}=f_{1} \vartheta
$$

here, $\quad f$ indicates the image and $\mathrm{V}$ denotes the speckle noise. $\vartheta=\left\{\vartheta_{1}, \vartheta_{2}, \ldots \ldots . . \vartheta_{n}\right\}$ is a unit mean random field.

Output is shown by the following equation:

$$
\hat{I}_{S} \sum_{p \in \eta s} m_{p} I_{p}
$$

Where, $m_{p}=\exp \left(-K C_{s}^{2} d_{s, p}\right) / \sum_{p \in 1} \exp \left(-K C_{s}^{2} d_{s, p}\right)(3)$ and

$$
d s, p=\sqrt{\left(i-i_{p}\right)^{2}+\left(j-j_{p}\right)^{2}}
$$


Where, $\mathrm{K}$ is nothing but the damping factor, $(\mathrm{i}, \mathrm{j})$ are the grid coordinates of pixel s, and (ip, jp) are the grid coordinates of pixel $\mathrm{p}$. The factor, $\mathrm{K}$, is selected bearing in mind, when $\mathrm{KC} 2 \mathrm{~s}$ approaches zero in a homogenous region, it yields the mean filter output; in contrast, at an edge, $\mathrm{KC} 2 \mathrm{~s}$ becomes so large that filtering is inhibited completely and is thus, rendered useless. From the above formula (3) and (4), Frost filter is not a directional filter. It is an isotropic diffusion filter. For approving this point choose the filter window as $w s=\{(i-$ $1, j),(i, j-1),(i+1, j),(i, j+1)\}$ at an interior site $(i, j)$, then according to (4), all the four values of $d$ in ws are the same. Thus, the Frost filter has no such system which allows the enhancement of edges or to feature structures within a window. In order to evaluate the results of filter quantitatively, the following four factors are defined and evaluated as:

$\sum_{M S E}=(1 / k)(\hat{S} i-S i)^{2}$

where, $\hat{S} i, S i$ represent the de noised and original images, respectively. $k$ represents the image size.

$P S N R=10 * \log _{10}\left(255^{2} / M S E_{)}\right.$

$S / M S E=10 * \log _{10}\left(\sum_{i=1}^{k} S i^{2} / \sum_{i=1}^{k}(\hat{S} i-S i)\right)$

FOM $=\frac{1}{\max \left\{\hat{N}, N_{\text {ideal }}\right.} \sum_{i=1}^{\hat{N}} \frac{1}{1+d i^{2} \alpha}$

where $\hat{N}_{, N_{\text {ideal }}}$ represent the number of detected and ideal edge pixels, respectively. ' $d_{i}$ ' is the Euclidean distance between the $i$ th edge pixel which is detected and the nearest edge pixel, and $\alpha$ is a constant, which typically takes the value of $1 / 9$. FOM lies in the range of 0 and 1 , with unity for ideal edge detection.

Mean $M=$ sum of $x$ values $/ N$

where $N$ is number of values

Standard Deviation $s=\sqrt{\frac{\sum(x-M)^{2}}{n-1}}$

where $M$ is mean.

Let's take the filter window to be $\eta_{s}=\bar{\eta}_{s}$. Thus, we have

from (4) that $d_{s, p}=1 \forall p \in \bar{\eta}_{S}$, which implies that $m_{p}=$ 1

$\overline{|\bar{\eta} s|}$ for any $p \in \bar{\eta}_{s}$. The Frost filter (2) is reduced, in this case, to

$\hat{I i, j=} \frac{1}{|\bar{\eta} s|} \sum_{p \in \bar{\eta} s_{s} I p} \frac{1}{|\bar{\eta} s|} \sum_{p \in \bar{\eta} S_{s}}(I p-I i, j)$

$$
=I_{i, j}+\frac{1}{|\bar{\eta} s|} \nabla^{2} I_{i, j}
$$

which is in resemblance with the form of the isotropic diffusion update function with $\Delta t=1$. If we are to reassign weights in (2) such that

$\hat{I} i, j=\frac{M_{i+1, j} I_{i+1, j}+M_{i, j} I_{i-1, j}+M_{i, j+1} I_{i, j+1}+M_{i, j} I_{i, j-1}}{M_{i+1, j}+M_{i, j}+M_{i, j+1}+M_{i, j}}$

$\hat{I} i, j$
$\frac{M_{i+1, j} I_{i+1, j}+M_{i, j} I_{i-1, j}+M_{i, j+1} I_{i, j+1}+M_{i, j} I_{i, j-1}}{M_{i+1, j}+M_{i, j}+M_{i, j+1}+M_{i, j}}$
$-I_{i, j}$

$=I_{i, j}+\frac{K_{0}^{\prime}}{\left|\bar{\eta}_{S}\right|} \operatorname{div[Mi,j} \nabla I i, j_{]}$

where $M_{i, j}=\exp \left(-K C^{2} i, j\right)$, we can recognize (26) as shown in (27), where $K_{0}^{\prime}=\left(\left(M_{i+l, j}+M_{i, j+l}+2 M_{i, j}\right) / 4\right)^{-1}$.

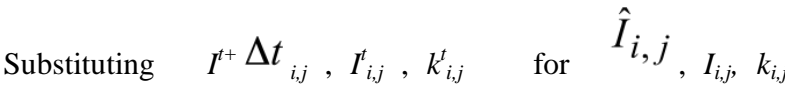
respectively, and letting $\Delta t=K^{\prime}{ }_{0}$ (its value is between 0 and 1), we show that (27) is in the form of (19), where $\Delta t$ is space-and-time varying.

\subsection{Lee Filter and Diffusion}

As mentioned above, the Lee filter is primarily designed keeping in mind the aim of eliminating speckle noise. It does not do so at the cost of the edges and point features in radar imagery and seeks to preserve these while still eliminating the speckle noise. The filter incorporates both, a linear speckle noise model and the Minimum Mean Square Error (MMSE) design approach, to produce the enhanced data, which is in accordance with:

$$
\hat{I}_{s}=\bar{I} s+k_{s}\left(I s-\bar{I}_{s}\right)
$$

Where, $\bar{I} s$ is the mean/average value of the intensity within the filter window $\eta^{s} ; k_{s}$ is the adaptive filter coefficient determined by:

$k_{s}=1-C_{u}^{2} / C_{s}^{2}$

Here,

$\left.C s^{2}=\left(1 / \mid \eta_{s}\right)\right) \sum_{p \oplus \eta}(I p-\bar{I} s)^{2} /(I p-\bar{I} s)^{2}$

and $C_{u}{ }^{2}$ is a constant for a given image and can be determined by either

$C_{u}^{2}=1 / E N L$ 
or $\quad C_{u}{ }^{2}=\frac{\operatorname{var}\left(z^{\prime}\right)}{\left(\bar{z}^{\prime}\right)^{2}}$

Where, ENL is the effective number of looks of the noisy image. This factor is used to derive noise variance. By adjusting ENL, the user can control the amount of smoothing applied to the image. Theoretically, the correct value of ENL should be the effective number of looks of the radar image. It should be near to the actual number of looks, but may vary if the image has undergone re sampling. A smaller value leads to more smoothing; a larger value preserves more image features. The ENL factor is related to the radiometric resolution of the image.

Anisotropic Diffusion:

$\left\{\frac{\partial I}{\partial t}=\operatorname{div}[c(|\nabla I|) \nabla I]\right.$

$I(t=0)=I_{0}$

$c(x)=\frac{1}{1+(x / k)^{2}}$

and $c(x)=\exp \left[-\left(x / k^{2}\right)\right]$

where, $k$ is an edge magnitude factor.

In the anisotropic diffusion method, the gradient magnitude is used in order to detect an edge or boundary as a step

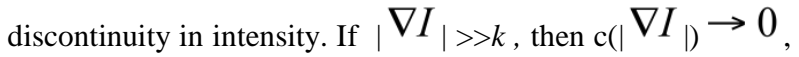
and we have an all pass filter; if $\mid \nabla I_{\mid<<k}$, then $\mathrm{c}\left(\mid \nabla_{I \mid}\right)$ $\rightarrow 1$, and we achieve isotropic diffusion (Gaussian filtering).

A discrete form of (16) can be represented as

$I_{\mathrm{s}}{ }^{t+\Delta t={ }_{I_{\mathrm{s}}{ }^{t}}}+\frac{\Delta t}{\left|\bar{\eta}_{s}\right|} \sum_{p \in \bar{\eta}_{s}} c\left(\nabla I^{t} s, p\right) \nabla I^{t} s, p$

Where, $I_{s}^{t}$ is the discretely sampled image, $s$ denotes the pixel position with respect to a discrete two dimensional (2-D) grid, $\Delta t$ represents the time step size, $\bar{\eta}_{s}$ denotes the spatial neighborhood of pixel $s, \mid \bar{\eta}_{s}$ is the number of pixels in the window (usually four, except at the image boundaries), and $\nabla I_{s, p}^{t}=I_{p}^{t}-I_{s}^{t}, \forall p \in \bar{\eta}_{s}$

Anisotropic diffusion helps smooth interior regions and helps preserve edges. In these categories, there are a vast number of applications. Another example is that of dealing with additive noise. The Lee filter generally works in a 7 by 7 window. Let us $\eta_{s}=\bar{\eta}_{s} \equiv\{(i-1, j),(i, j-1)(i+1, j),(i, j+1)\}$ at an interior site $s=(i, j)$. We can,thus, choose to depict the special case of the Lee filter with window, $\bar{\eta} S$, in the following form: $\hat{I}_{i, j}=I_{i, j}+\left(1-k_{i, j}\right)\left(\bar{I}_{i, j}-I_{i, j}\right)$

$\left(\frac{1}{\left|\overline{\eta_{s}}\right|} \sum_{p \in \bar{\eta}_{s}} I p-I i, j\right)$

$\begin{aligned}= & I_{i,} j_{j}+\left(1-k_{i, j}\right) \\ \left(\frac{1}{\left|\bar{\eta}_{s}\right|} \sum_{p \in \bar{\eta} s} I p-I i, j\right) & \end{aligned}$

$$
=I_{i, j}+\left(1-k_{i, j}\right) \frac{1}{\left|\overline{\eta_{s}}\right|} \nabla_{2} I i, j
$$

where $\left.\left.\right|^{\bar{\eta}_{s}}\right|_{\mid(=4)}$ is the number of pixels in the window.

Substituting $\quad I_{i, j}^{t+1}, I^{t} i, j, k_{i, j}^{t}$ for $\hat{I} i, j, I i, j, k i, j$ in (20), respectively, we obtain $\quad I_{i, j}{ }^{t+1}=I^{t} i, j+\left(1-k_{i, j}^{t}\right) \frac{1}{\left|\overline{\eta_{S}}\right|}$ 2

$\nabla \quad I^{t} i, j$

Comparing (21) with (19) and noting the equality

$$
\begin{aligned}
\nabla^{2} I_{i, j}=I_{i+1, j}+I_{i-1, j}+I_{i, j+1}+I_{i, j-1}-4 I_{i, j} \\
\\
=\sum_{p \in \bar{\eta} s}(I p-I i, j)
\end{aligned}
$$

It is important to note that the form represented in (21) is a special case of (19), in that, $c($ ) can be taken out of the summation and $\Delta t=1$. In simple words, the Lee filter is similar to that of a discrete isotropic diffusion equation. Furthermore, it is compelling to note the effect of assigning slightly varying weights to the four directional differences in (21), such that,

$$
\begin{aligned}
& \hat{I i, j}=I_{i, j}+\frac{1}{\left|\bar{\eta}_{S}\right|}\left[\left(1-k_{i+1, j}\right)\left(I_{i+1, j}-I_{i, j}\right)+\left(1-k_{i, j}\right)\left(I_{i+1, j}-\right.\right. \\
& \left.I_{i, j}\right) \\
& \left.+\left(1-k_{i, j+1}\right)\left(I_{i, j+1}-I_{i, j}\right)+\left(1-k_{i, j}\right)\left(I_{i, j-1}-I_{i, j}\right)\right] .
\end{aligned}
$$

In fact, (23) reduces to

$$
\hat{I} i, j_{=} I_{i, j_{+}} \frac{1}{\left|\overline{\eta_{s}}\right|} \operatorname{div[(1-k_{i,j})} \nabla I_{i, j_{]}} .
$$

with the recommended variable substitutions in (24), it is easily recognizable that (24) is in a form analogous to that of anisotropic diffusion [as in (19)], where, $\Delta t=1$.

Consequently, with (20), the diffusion assumes a sort of edge directional sensitivity. 


\subsection{SRAD Filter}

SRAD's PDE is expressed as follows:

$$
\begin{aligned}
& \left\{\frac{\partial I(u, v ; t)}{\partial t}=\operatorname{div}[c(q) \nabla I(u, v, ; t)]\right. \\
& I(u, v ; 0)=I_{0}(u, v),\left.\left(\frac{\partial I(u, v, ; t)}{\partial \bar{\eta}}\right)\right|_{\partial \Omega}=0
\end{aligned}
$$

where $\nabla$ is the gradient operator; div represents the divergence operator; $I(u, v ; t)$ the image's intensity estimated at position $u, v$, at time $t$;

The diffusivity function of SRAD is given as:

$c(q)=\frac{1}{1+\frac{\left[q^{2}(u, v ; t)-q_{0}^{2}(t)\right]}{\left[q_{0}^{2}(t)\left(1+q_{o}^{2}(t)\right)\right]}}$

where $q(u, v ; t)$ defined as -

$q=\sqrt{\frac{(1 / 2)\left(|\nabla I| / I^{2}-\left(1 / 4^{2}\right)\left(\nabla^{2} I\right) / I\right)^{2}}{\left[1+(1 / 4)\left(\nabla^{2} I / I\right)\right]^{2}}}$

and $q$, can be described as -

$$
q_{0}(t)=\frac{\sqrt{\operatorname{var}[z(t)]}}{z(\bar{t})}
$$

So, automatic determination of $q_{o}(t)$ is desired in real application to eliminate heuristic factor choice.

To begin with, we assert that $\mathrm{q}_{\mathrm{o}}(\mathrm{t})$ can be approximated by

$q_{o}(t)=q_{o} \exp \left[-{ }^{\rho} t\right.$

where, $\rho$ is a constant, and $q_{o}$, the speckle coefficient of variation in the observed image. Considering a fully developed speckle, $q_{o}=1$ for ultrasound intensity data (without compounding) and $q_{o}=1 / \sqrt{N}$ for $N$-look SAR intensity data. For partial correlated speckle, $q_{o}$ is less than unity. We can thus, give the derivation of (32). Diffusion remains isotropic in an area with similar properties. On incorporating the discrete isotropic diffusion update, we get

$I_{i, j}^{t+1}=I_{i, j}^{t}+\frac{\frac{\Delta t}{4}}{\left(I_{i+1, j}^{t}+I_{i-1, j}^{t}+I_{i, j+1}^{t}+I_{i, j-1}^{t}-4 I_{i, j}^{t}\right)}$

The differential equation is numerically solved by using iterative Jacobi method. When a time step size, $\Delta t$, which tends to zero is taken along with a small spatial step size of $h$ in $x$ and $y$ directions, we are free to discretise the time and space coordinates as follows:

$$
\begin{array}{rlrl}
t=n \Delta t, & n=0,1,2, \ldots \ldots \ldots . . & & \\
x & =i h, & i=0,1,2, \ldots \ldots, M-1 \\
y & =j h, & j=0,1,2, \ldots \ldots, N-1
\end{array}
$$

where, $M h * N h$ is the size of the image support. Let $I_{i, j}^{n}=I(i h$ , jh, ${ }_{n} \Delta t$ ). Moving forward, a three- stage approach is employed to calculate the right hand side of the SRAD PDE. In the first stage, the derivative and Laplacian approximations are computed as

$\nabla_{R} I^{n} i, j=\left[\frac{I^{n} i+1, j-I^{n} i, j+1}{h}, \frac{I^{n} i, j+1-I^{n} i, j}{h}\right]_{(34)}$

$\nabla_{L}{ }^{n} i, j=\left[\frac{I^{n} i, j-I^{n} i-1, j}{h}, \frac{I^{n} i, j-I^{n} i, j-1}{h}\right]_{(35)}$ $2 n$ $\nabla I_{i, j}=$

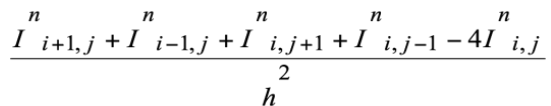

with symmetric boundary conditions

$I_{-1, j}^{n}=I_{0, j}^{n}, I_{M, j}^{n}=I_{M-1, j}^{n}$,

$j=0,1,2, \ldots \ldots ., N-1$

$I^{n}{ }_{i,-1}=I_{i, 0}^{n}, \quad I_{i, N}^{n}=I_{i, N-1}^{n}$

$i=0,1,2, \ldots \ldots \ldots, M-1$

In the second stage, we proceed to compute the diffusion coefficient $c(q)$ according to

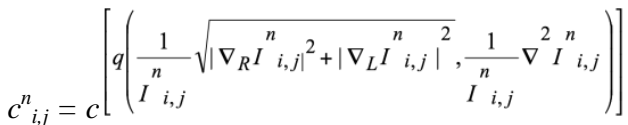

In the third stage, we compute the divergence of $c(.) \nabla I$ which is essential for the SRAD PDE in (28), as

$$
\begin{aligned}
& \frac{1}{2} \\
d^{n}{ }_{i, j}= & h^{[}\left[c^{n}{ }_{i+1, j}\left(I^{n}{ }_{i+1 j}-I_{i, j}^{n}\right)+c^{n}{ }_{i, j}\left(I^{n}{ }_{i-1 j}-I_{i, j}^{n}\right)\right. \\
+ & \left.c^{n}{ }_{i, j+1}\left(I_{i, j+1}^{n}-I_{i, j}^{n}\right)+c^{n}{ }_{i, j}\left(I_{i, j-1}^{n}-I_{i, j}^{n}\right)\right]
\end{aligned}
$$

with symmetric boundary conditions

$$
\begin{aligned}
& d^{n}{ }_{-1, j}=d^{n}{ }_{0, j}, \quad d^{n}{ }_{M, j} \quad=\quad d^{n}{ }_{M-1, j} \\
& j=0,1,2, \ldots \ldots \ldots, N-1 \\
& d_{\mathrm{i},-1}^{n}=d_{i, 0}^{n}, \quad d^{n}{ }_{i, N}=d^{n}{ }_{i, N-1}, \\
& i=0,1,2, \ldots \ldots \ldots, M-1
\end{aligned}
$$

To sum up, by the approximation of time derivative with respect to forward differencing, the numerical approximation to the differential equation is stipulated as

$I_{i, j}^{n+1}=I_{i, j}^{n}+\frac{\Delta t}{4} d_{i, j}^{n}$

Equation (43) represents the SRAD update function. Now that the SRAD update function is obtained, we can proceed to demonstrate the proficiency of SRAD in terms of preserving the intra-region mean as well as edge positions and reducing 
the intra-region variance. In numerical implementation, we select $h=1$ and $\Delta t=0.05$; furthermore, as the diffusion coefficient tends to zero but never actually reaches it at any given edge in a digital image, as an alternative, we may set $c^{n}{ }_{i, j}$ to zero if it is less than a lower threshold $T$, to halt diffusion across main edges

\section{RESULTS}

The performance of the Frost filter, Lee filter and SRAD filter is studied with operations on ultrasound images using MATLAB software. The peak signal to noise ratio (PSNR) and Pratt's figure of Merit (FOM) of each ultrasound image has been calculated.

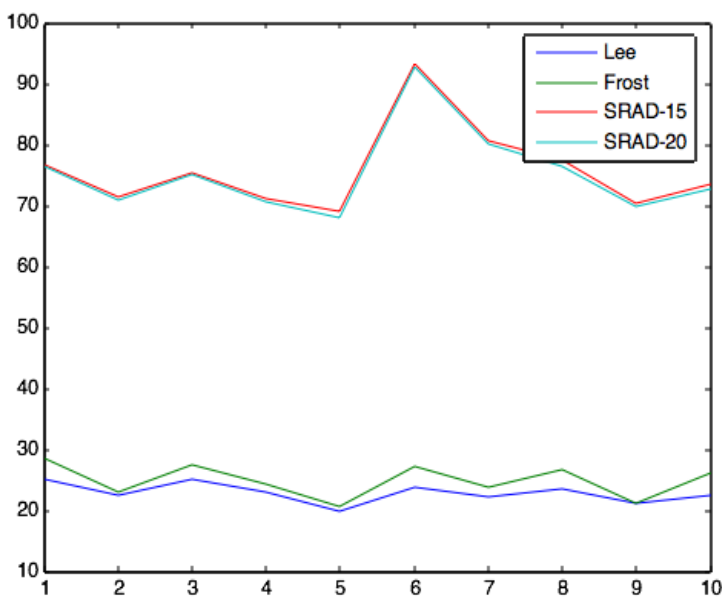

Fig1. Comparison chart of PSNR for ultrasound image corrupted by speckle noise.

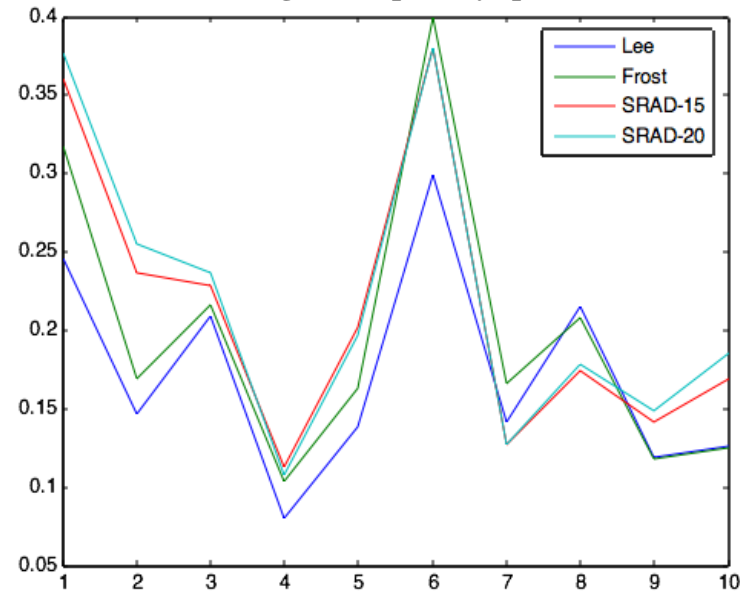

Fig 2. Comparison chart of FOM for ultrasound image corrupted by speckle noise.

\section{CONCLUSION}

To conclude, a small, negligent threshold value will provide all the noisy coefficients but the final image may still contain speckle. A larger threshold value sets a higher number of coefficients to zero which smoothens the signal, destroying details and the resultant image may cause blur and artifacts. Hence, the optimum threshold value has to be calculated and simulation to be tuned accordingly, which adapts to different sub band characteristics.

\section{FUTURE WORK}

Our goal now is to develop a new method that will handle this problem and will provide more enhanced images by retaining its original characteristics as the number of iterations increase. The key concept for such development of the new method lies in analyzing the quality of image which is based on the FOM values. FOM value ranges between 0 and 1 . Higher the FOM value in the range of 0 to 1 , better the quality of the ultrasound image. So the focus will be on designing a method that filters the unwanted noise and enhances the quality of image based upon the FOM value.

\section{REFERENCES}

[1] B.Yoo and T. Nishimura, "A Study of Ultrasound Image Enhancement Using Adaptive Speckle Reducing Anisotropic Diffusion," presented at IEEE International Symposium on Industrial Electronics ,pp.: 581-585,2009.

[2] Y. Yu and S.T. Acton, "Speckle reducing anisotropic diffusion, "IEEE Trans. Image Process., vol. 11, no.11,pp. 1260-1270,Nov. 2002.

[3] S. T.Acton, "Deconvolutional Speckle Reducing Anisotropic Diffusion," presented at Image Processing, 2005, ICIP 2005. IEEE International Conference on, 2005.

[4] X. Zhi, T. Wang, "An Anisotropic Diffusion Filter for Ultrasonic Speckle Reduction," presented at Institution of Engineering and Technology 2008.

[5] "Matlab", www.mathworks.com/products/matlab/, November 24,2014.

\section{AUTHOR PROFILE}

\section{Paulami Shah}

Paulami Shah is an assistant professor in the computer engineering department of Mukesh Patel School of Technology Management \& Engineering, NMIMS University. Areas of Interests: Image Processing, Big Data Analytics, Web Technology \& Programming.

\section{Saurabh Malgaonkar}

Saurabh Malgaonkar is an assistant professor in the computer engineering department of Mukesh Patel School of Technology Management \& Engineering, NMIMS University. Areas of Interest: Networks, Distributed Computing.

\section{Tanay Parekhji}

Tanay Parekhji is currently pursuing the final year of Bachelor's in Computer Science and Engineering from NMIMS's Mukesh Patel School of Technology Management and Engineering, NMIMS University. His area of interests are Artificial Intelligence, Operating Systems and Computer Animation Physics.

\section{Rahul Chavan}

Rahul Chavan is currently pursuing a Bachelor's degree in Computer Science from NMIMS University, Mumbai. His areas of interest include Artificial Intelligence, Software Development and Coding. Rahul Chavan is currently pursuing a Bachelor's degree in Computer Science from NMIMS University, Mumbai.His areas of interest include Artificial Intelligence, Software Development and Coding. 